\title{
Pulmonary function in patients with Amyotrophic Lateral Sclerosis at disease onset
}

\author{
B. Chandrasoma1, D. Balfe ${ }^{1}$, T. Naik2 , A. Elsayegh1, \\ M. Lewis ${ }^{2}$, Z. Mosenifar ${ }^{1}$
}

ABSTRACT: Pulmonary function in patients with Amyotrophic Lateral Sclerosis at disease onset. B. Chandrasoma, D. Balfe, T. Naik, A. Elsayegh, M. Lewis, Z. Mosenifar.

Background. Amyotrophic Lateral Sclerosis (ALS) is a progressive neurodegenerative disorder affecting both the upper and lower motor neurons. Deteriorating pulmonary function as a reflection of progressive respiratory muscle weakness is a common feature, accounting for the majority of deaths. The aim of the study was to describe a trend in initial pulmonary function tests (PFT) of Amyotrophic Lateral Sclerosis (ALS) patients, in addition, differentiating between the types of disease onset, bulbar, limb muscle, and a combination.

Methods. Initial PFT were gathered from 32 consecutive patients in our clinic with the diagnosis of ALS, they were categorized by the type of disease onset. Values obtained were referenced to the $95 \%$ confidence limits for normality.
Results. There was evidence of significant reductions in both the $\mathrm{FEV}_{1}(64.7 \%$ predicted) and FVC $(61.2 \%)$, with preservation of the $\mathrm{FEV}_{1} / \mathrm{FVC}(\mathbf{8 1 . 7 \%})$. The MVV was significantly reduced $(43 \%)$. Total lung capacity was 93.2\%, the residual volumes was increased at $145.7 \%$. Subgroup analysis failed to show significant differences between types of disease onset. In the bulbar onset group (versus the limb group) there was a trend for the MVV to be further reduced $(p=0.15)$ and the $R V$ to be higher (157.4\% versus $135.9 \%, P=0.24)$.

Conclusions. ALS is a devastating disease that invariably leads to respiratory failure. Abnormal spirometric variables such as the FVC and MVV, likely reflect inspiratory muscle weakness and increased $\mathrm{RV}$ likely reflect expiratory muscle weakness. The type of disease onset did not result in a different pattern of PFT abnormalities. Monaldi Arch Chest Dis 2012; 77: 3-4, 129-133.

Keywords: Amyotrophic Lateral Sclerosis, Pulmonary function test, Restrictive lung disorder.

Correspondence: Dr. David Balfe, Division of Pulmonary/Critical Care Medicine, Cedars-Sinai Medical Center, 8700 Beverly Blvd \#6732, Los Angeles, CA 90048, U.S.A.; e-mail: David.Balfe@cshs.org

\section{Introduction}

Amyotrophic lateral sclerosis (ALS) is a devastating progressive neurodegenerative disorder characterized by degeneration of both upper and lower motor neurons [1]. Lower motor neurons involved include anterior horn cells in the spinal cord and cranial nerve homologues in the brainstem, while upper motor neuron degeneration occurs in motor cortex neurons and their descending tracts (corticospinal). Depending on the predominant neuron groups involved, several patterns of disease may be evident in the early stages, such as predominantly bulbar or limb muscle involvement or both [2]. Limb muscle and /or bulbar muscle weakness and dysfunction is progressive, culminating in the later stages with progressive respiratory muscle weakness and eventually ventilatory failure, which is the most common cause of death. The average life expectancy has been increasing with the use of respiratory interventions such as tracheostomy in addition to noninvasive and invasive ventilatory support. Despite this however, the current life expectancy remains less than 5 years in $80 \%$, from the time of diagnosis and less than 3 years in $50 \%$ of patients [3-5].
Since patients with ALS often do not have respiratory complaints upon their initial presentation, pulmonary function testing is commonly obtained at the time of respiratory symptom onset or when a clear diagnosis is firmly established in the absence of symptoms, which is the usual scenario. In one study for example, only $11 \%$ of patients exhibited pulmonary symptoms at the time of the initial diagnosis of ALS [6]. There is some controversy in the literature as to whether patients presenting with predominant bulbar disease, have worse subclinical respiratory muscle involvement, than do those presenting with predominantly limb muscle weakness [6-9]. Impairment in pulmonary function tests in the absence of symptoms may be of value in predicting subsequent disease course and prognosis. The aim of this study was thus to examine possible differences in spirometry, lung volumes and diffusing capacity between patient groups with predominantly bulbar or limb muscle presentations, tested on initial diagnosis or with first onset of pulmonary symptoms.

\section{Methods}

This study was conducted at Cedars-Sinai Medical Center in Los Angeles, California. The 
protocol was approved by the Cedars Sinai Institutional Review Board, approval number PR00020196. A chart review of initial pulmonary function testing for patients with known ALS from our clinic was retrospectively analyzed.

Pulmonary function tests (PFTs) were gathered from 32 consecutive patients in our pulmonary clinic with an established diagnosis of ALS. These patients were typically referred for pulmonary function testing and pulmonary evaluation once the diagnosis was established or when they had developed early respiratory complaints. PFTs were commonly followed to document progression of disease, however for the purposes of this study, only those initial tests for each patient were included in our data set.

The data included in the analysis were: forced vital capacity (FVC), forced expired volume in one second $\left(\mathrm{FEV}_{1}\right)$, the ratio of $\mathrm{FEV}_{1} / \mathrm{FVC}$, maximum voluntary ventilation (MVV), total lung capacity (TLC), residual volume (RV), and diffusing capacity (DLCO). Means and standard deviations were determined for each parameter. The prediction equations utilized were as follows:

FVC: $\quad($ male $)=0.119 *$ height $($ inches $)$

$-0.022 *$ age (years) -2.815

$($ female $)=0.093 *$ height $($ inches $)$

$-0.022 *$ age (years) -1.924

$\mathrm{FEV}_{1}: \quad($ male $)=0.085^{*}$ height $($ inches $)$

$-0.031 *$ age $($ years $)-0.879$

$($ female $)=0.027 *$ height $($ inches $)$

$-0.027^{*}$ age $($ years $)-0.525$

MVV: $\quad($ male $)=3.39 *$ height (inches)

$-1.26^{*}$ age (years) -21.40

$($ female $)=2.05 *$ height (inches)

$-0.57 *$ age (years) -5.50

$\mathrm{RV}: \quad($ male $)=0.049 *$ height (inches)

$+0.012 *$ age $($ years $)-2.240$

$($ female $)=0.081 *$ height (inches)

$+0.009 *$ age (years) -3.900

DLCO: $($ male $)=0.148 *$ height (inches)

$-0.229 *$ age $($ years $)+12.911$

$($ female $)=0.407 *$ height $($ inches $)$

$-0.111 *$ age (years) +2.24

The data was then further categorized by the clinical type of onset of disease: namely, predominantly bulbar disease, predominantly limb muscle weakness, or a combination of both manifestations. This information was extracted from chart review of the patient's initial neurologic evaluation. The mean values for each component of the pulmonary testing were used to compare the subtypes in terms of their initial onset of disease. In our cohort of patients, PFTs were ordered mostly following respiratory symptom onset, and thus these initial PFTs were used to signify their pulmonary function at that time for the entire cohort as well as the subtypes specified above.

\section{Statistical Analysis}

Numerical variables were summarized by Mean \pm SD. Differences across the groups were assessed by ANOVA and the Kruskal-Wallis test. Categorical variables were summarized by frequency and percent. Differences across the groups were assessed by the Fisher exact test. The 0.05 significance level was used for all significance tests. SAS version 9.1.3 (SAS Institute, Cary, North Carolina) was used for all statistical calculations.

\section{Results}

\section{Demographic Data}

Demographic details on gender, age at disease onset and body weight, are depicted in table 1. For all patients and the subgroups, there was a male predominance, and no significant difference in age or body weights.

\section{Patient Cohort PFTs}

From the global analysis derived from the mean values of all 32 patients, there were several notable observations. There was evidence of reduction in both the $\mathrm{FEV}_{1}(64.7 \pm 24.5 \%)$ and FVC $(61.2 \pm 21.1 \%)$ with normal $\mathrm{FEV}_{1} / \mathrm{FVC}$ ratio $(81.7$ $\pm 10.5 \%)$. The total lung capacity remained normal $(93.2 \pm 16.6 \%)$, however the residual volume was markedly increased $(145.7 \pm 40.2 \%)$. The DLCO was mildly reduced $(71.5 \pm 18.5 \%)$, but proved to be appropriate with correction for the alveolar volume.

\section{Subgroup PFT Analysis}

The Subgroup data are depicted in table 2 and figure 1. Analysis across the subgroups revealed reduced FVC and $\mathrm{FEV}_{1}$ throughout, with a tendency for lower values in the bulbar group. $\mathrm{FEV}_{1} / \mathrm{FVC}$ ratio was normal in all subgroups. While this suggests a restrictive ventilatory defect, the total lung capacity was found to be normal in all subgroups, the MVV was reduced in all subgroups. There was a trend towards more significant MVV reduction in the bulbar onset group, but this did not reach statistical significance $(\mathrm{p}=0.15$ versus the limb group). The RV (percent predicted) was also noted to be higher among the bulbar onset patients when compared to the limb onset patients $(157.4 \%$ predicted versus $135.9 \%$ predicted respectively), but again failed to achieve statistical significance $(\mathrm{p}=0.24)$. For other parameters, values were similar across the groups.

\section{Discussion}

Amyotrophic lateral sclerosis is a devastating disease of progressive paralysis and ultimately respiratory failure leading to death, as highlighted in the introduction $[4,5,10]$. Pulmonary function testing has been evaluated in this patient popula- 
Table 1. - Demographic data of ALS patients

\begin{tabular}{lccccc}
\hline & All Patients & Bulbar & Limb & Combined & P value \\
\hline Male (Female) & $22(10)$ & $7(5)$ & $8(4)$ & $7(1)$ & \\
\hline Male (Female) $\%$ & $68.8(31.2)$ & $58.3(41.7)$ & $66.7(33.3)$ & $87.5(12.5)$ & 0.5 \\
\hline Weight (lbs) \pm SD & $156.0 \pm 35.6$ & $150.6 \pm 37.7$ & $160.3 \pm 33.2$ & $157.5 \pm 39.3$ & 0.80 \\
\hline Age (years) \pm SD & $60.1 \pm 12.3$ & $59.1 \pm 12.5$ & $61.2 \pm 11.4$ & $59.9 \pm 14.6$ & 0.92 \\
\hline
\end{tabular}

Data is presented as means \pm standard deviations. P-values were calculated using the Anova P test for numerical variables and Fisher exact test for categorical variables.

tion in the past with the most characteristic changes being reduction in the FVC [11]. Our study is in keeping with several others, in that significant decrements in spirometric values can be present early on in the disease process, often when patients exhibit no respiratory complaints [12]. Our study sought to further characterize whether patients presenting with predominantly bulbar disease, predominantly limb muscle weakness, or a combination of both manifestations exhibit differences in commonly performed PFTs.

\section{Demographics}

The mean ages of our subjects were slightly higher than reported previously $[8,9,12,13]$. Our cohort showed a male predominance $(68.8 \%$, male to female ratio of $2.2: 1$ ) as reported in other studies [5, 12,13]. In a study evaluating 1034 patients with ALS [13], the male to female ratio was 1.9:1, only $16.6 \%$ presented with primary bulbar features. By contrast, in the present study, the percentage of patients with predominantly bulbar onset was higher $(37.5 \%)$ similar to several other reports [6-8].

\section{Pulmonary Function Studies}

Spirometry: The most notable findings for the entire cohort were a reduction in $\mathrm{FEV}_{1}$ and $\mathrm{FVC}$ with a preserved $\mathrm{FEV}_{1} / \mathrm{FVC}$ ratio, and a reduced MVV. A reduction in $\mathrm{FEV}_{1}$ and $\mathrm{FVC}$ with preserved $\mathrm{FEV}_{1} / \mathrm{FVC}$ ratio suggests a restrictive ventilatory defect, however was not confirmed by a significant reduction in TLC, in our study. Prior studies in which only spirometry was employed, may have over called a true restrictive defect by ATS criteria. It is also of interest that while the $\mathrm{FEV}_{1} / \mathrm{FVC}$ ratio was within normal limits for the group as a whole, the variances were wide, several patients did show elevated ratios. While this apparent increased recoil would not be expected with pure inspiratory muscle weakness, the presence of possible recurrent aspiration with interstitial changes and/or hypoventilation-induced micro atelectasis may well explain such findings. A reduced MVV has been associated with reduced respiratory muscle strength, this may have been responsible for the reduced MVV that was noted.
Lung Volumes: TLC for the cohort of patients fell within the normal $95 \%$ confidence limits. Thus a restrictive ventilatory defect was not by definition evident in these patients. Note as the TLC was measured by helium dilution in half the patients, TLC was underestimated which further underscores the absence of a restrictive ventilatory defect (see table 2). The significant elevation in RV is notable, and goes along with concomitant expiratory muscle weakness with inability to generate a normal ERV. Expiratory muscle weakness goes along with generalized skeletal muscle involvement in patients with ALS. The clinical significance is a poor cough ability which may predispose to retained secretions, atelectasis and hypostatic pneumonia [5].

Diffusing Capacity: The DLCO was slightly reduced in this cohort of patients. Again, variances were wide precluding making strong inferences on the mechanism/s for the group as a whole. However, one wonders whether microatelectasis with reduction in surface area in part played a role in some instances. Of note, DLCO/VA fell within normal confidence limits.

\section{Bulbar Onset versus Limb Muscle Onset}

What is the impact of bulbar vs. limb muscle onset as it pertains to lung function tests [6-9]? In one study, FVC was lower in the bulbar group compared to the limb onset patients [9], while no differences were reported for these subgroups by Talakad and coworkers [6]. In 2 other studies, maximum inspiratory (PI $\max$ ) and expiratory (PE max) mouth pressures were significantly lower in the bulbar patients [8], while FVC was predictive in the bulbar onset group of subsequent respiratory insufficiency, but not in the spinal-onset group [7].

In the present study, spirometric parameters were generally similar across the groups. There was a trend however for the MVV to be further reduced and the RV to be further augmented in the bulbar onset group (see table 2). While it should be noted that these differences did not reach statistical significance, one wonders whether this reflects the low $n$ per group along with the wide variances noted. Thus our data tends to support a greater impact on PFTs in the bulbar patients, as reported by oth- 
Table 2. - Comparison of pulmonary function tests between types of onset of ALS

\begin{tabular}{|c|c|c|c|}
\hline Para-meters & $\begin{array}{c}\text { Bulbar onset } \\
(\% \text { predicted } \pm \text { SD })\end{array}$ & $\begin{array}{c}\text { Limb onset } \\
(\% \text { predicted } \pm \text { SD })\end{array}$ & $\begin{array}{l}\text { Combined onset } \\
\text { (\% predicted } \pm \text { SD) }\end{array}$ \\
\hline FVC (L) & $59.5 \pm 18.2$ & $63.8 \pm 20.6$ & $60.0 \pm 27.6$ \\
\hline $\mathrm{FEV}_{1}(\mathrm{~L})$ & $63.3 \pm 23.3$ & $69.7 \pm 24.0$ & $59.5 \pm 28.6$ \\
\hline $\mathrm{FEV}_{1} / \mathrm{FVC}$ & $83.1 \pm 12.7$ & $83.2 \pm 4.5$ & $77.5 \pm 13.2$ \\
\hline MVV (L) & $39.8 \pm 7.2$ & $49.0 \pm 20.1$ & $42.3 \pm 21.7$ \\
\hline TLC (L) & $94.5 \pm 8.0$ & $90.6 \pm 18.3$ & $95.7 \pm 25.0$ \\
\hline $\mathrm{RV}(\mathrm{L})$ & $157.4 \pm 43.7$ & $135.9 \pm 35.2$ & $144.0 \pm 45.0$ \\
\hline $\mathrm{DLCO}(\mathrm{ml} \mathrm{CO} / \mathrm{min} / \mathrm{mmHg})$ & $70.8 \pm 15.6$ & $74.2 \pm 23.6$ & $68.3 \pm 15.9$ \\
\hline $\mathrm{DLCO} / \mathrm{VA}(\mathrm{CO} / \mathrm{min} / \mathrm{mmHg})$ & $107.4 \pm 29.7$ & $113.0 \pm 22.1$ & $93.7 \pm 19.7$ \\
\hline $\mathrm{FRC}(\mathrm{L})$ & $127.3 \pm 35.3$ & $120.5 \pm 33.3$ & $121.8 \pm 32.4$ \\
\hline Plethysmography & $136.0 \pm 42.0$ & $121.2 \pm 22.3$ & $159.5 \pm 16.3$ \\
\hline Helium dilution & $114.3 \pm 20.5$ & $119.8 \pm 42.6$ & $103.0 \pm 15.6$ \\
\hline
\end{tabular}

ers in the literature [6-8]. One cause for concern in making inferences for the bulbar patients, is the fact that their bulbar involvement may preclude these patients from keeping a tight mouth seal during pulmonary function maneuvers, which may alter their reliability.

\section{Clinical Implications}

Of major importance is the fact that in our series and that of others, PFTs performed early in the natural history of ALS are commonly abnormal, even in patients in whom pulmonary symptoms are absent $[6,12]$. Further, simple tests as the measurement of the FVC may have prognostic implications. For example, at disease onset, patients with a $\mathrm{FVC}<75 \%$ of predicted had a median survival of 2.9 years, compared to median survival of 4.08 years in those ALS patients with FVC $>75 \%$ of predicted at baseline [13]. FVC can also be used to follow the progress of disease. Expiratory muscle weakness appears to be an early phenomenon along with aberrations in spirometry in patients with ALS regardless of presentation. Development of a true restrictive ventilatory defect appears to be a later phenomenon as inspiratory muscle weakness progresses.

What other simple tests can also be employed? Sitting and supine FVC as measures of possible diaphragm weakness are examples. In one study, supine FVC was more predictive of 2 year survival than sitting FVC [14], in addition, inspiratory and expiratory mouth pressures can be employed. In this regard, Mendoza et al [15] reported that MIP, was more sensitive than FVC in predicting a

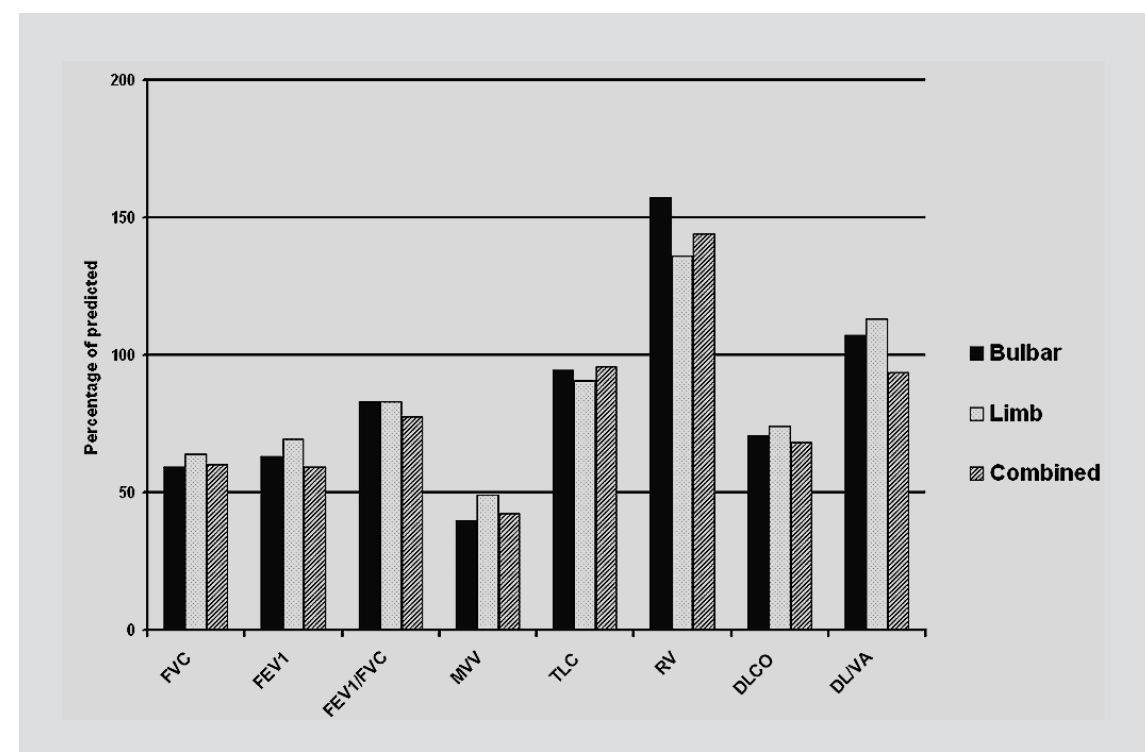

Fig. 1. - Comparison of percent predicted values for the components of pulmonary function testing. Abbreviations: Forced Vital Capacity (FVC); Forced Expiratory Volume in 1 second $\left(\mathrm{FEV}_{1}\right)$; Maximal Voluntary Ventilation (MVV); Total Lung Capacity (TLC); Residual Volume (RV); Diffusing Capacity for Carbon Monoxide (DLCO); Alveolar Volume (VA). 
threshold to consider non-invasive ventilation in the course of disease and reach this threshold 4-6.5 months earlier than the FVC threshold.

To conclude, our data reinforce early changes in PFTs at disease onset. Reduction in spirometric variable as described above and increment in RV are distinctive and of importance regarding current disease status and subsequent progression. The PFT aberrations are similar regardless of the clinical mode of disease presentation. Bulbar onset, however, may portend greater inspiratory and expiratory muscle weakness. Careful and serial follow up is mandatory as the disease progresses despite the use of agents such as the glutamate antagonist, riluzole [16], in patients in whom the diagnosis of ALS has not been considered or confirmed, a PFT pattern as described above may be useful in suggesting the presence of this devastating disease process.

Acknowledgements: Statistical assistance by James Mirocha, MS.

\section{References}

1. Rowland LP, Shneider NA. Amyotrophic lateral sclerosis. N Engl J Med 2001; 344: 1688-700.

2. Sathyaprabha TN, et al. Assessment of Pulmonary Function in Amyotrophic Lateral Sclerosis. Indian J Chest Dis Allied Sci 2009; 51: 87-91.

3. Wijesekera LC, Leigh PN. Amyotrophic lateral sclerosis. Orphanet Rare Dis 2009, 4: 3.

4. Bach JR. Amyotrophic Lateral Sclerosis: Prolongation of Life by Noninvasive Respiratory Aids. Chest 2002; 122; 92-98.

5. Mistry S, Lewis MI. Neuromuscular Respiratory Failure in the ICU: an Overview. In: Practical Pulmonary and Critical Care Medicine: Disease Management. Z Mohsenifar, PK, G SooHoo (eds.) Taylor and Francis. Volume 214, 221-285; 2006.
6. Talakad NS, Pradhan C, Nalini A, Thennarasu K, Raju TR. Assessment of pulmonary function in amyotrophic lateral sclerosis. Indian J Chest Dis Allied Sci 2009; 51: 87-91.

7. Pinto S, Turkman A, Pinto A, Swash M, de Carvalho M. Predicting respiratory insufficiency in amyotrophic lateral sclerosis: the role of phrenic nerve studies. Clin Neurophysiol 2009; 120: 941-6.

8. Pinto S, Pinto A, De Carvalho M. Do bulbar-onset amyotrophic lateral sclerosis patients have an earlier respiratory involvement than spinal-onset amyotrophic lateral sclerosis patients? Eura Medicophys 2007; 43: 505-9.

9. Ilzecka J, Stelmasiak Z, Balicka G. Respiratory function in amyotrophic lateral sclerosis. Neurol Sci 2003; 24: 288-9.

10. Fallat RJ, Jewitt B, Bass M, Kamm B, Norris FH Jr. Spirometry in amyotrophic lateral sclerosis. Arch Neurol 1979; 36: 74-80.

11. Schiffman PL, Belsh JM. Pulmonary function at diagnosis of amyotrophic lateral sclerosis. Rate of deterioration. Chest 1993: 103; 508-513.

12. Sathyaprabha TN, Pradhan C, Nalini A, Thennarasu K, Raju TR. Pulmonary function tests and diaphragmatic compound muscle action potential in patients with sporadic amyotrophic lateral sclerosis. Acta Neurol Scand 2010; 121: 400-5.

13. Czaplinski A, Yen AA, Appel SH. Forced vital capacity (FVC) as an indicator of survival and disease progression in an ALS clinic population. J Neurol Neurosurg Psychiatry 2006; 77: 390-2.

14. Baumann F, Henderson RD, Morrison SC, et al. Use of respiratory function tests to predict survival in amyotrophic lateral sclerosis. Amyotroph Lateral Scler 2010; 11: 194-202.

15. Mendoza M, Gelinas DF, Moore DH, Miller RG. A comparison of maximal inspiratory pressure and forced vital capacity as potential criteria for initiating non-invasive ventilation in amyotrophic lateral sclerosis. Amyotroph Lateral Scler 2007; 8: 106-11.

16. Bensimon G, Lacomblez L, Meininger V, ALS/Riluzole Study Group. A controlled trial of riluzole in amyotrophic lateral sclerosis. New Engl J Med 1994; 330: 585-591.

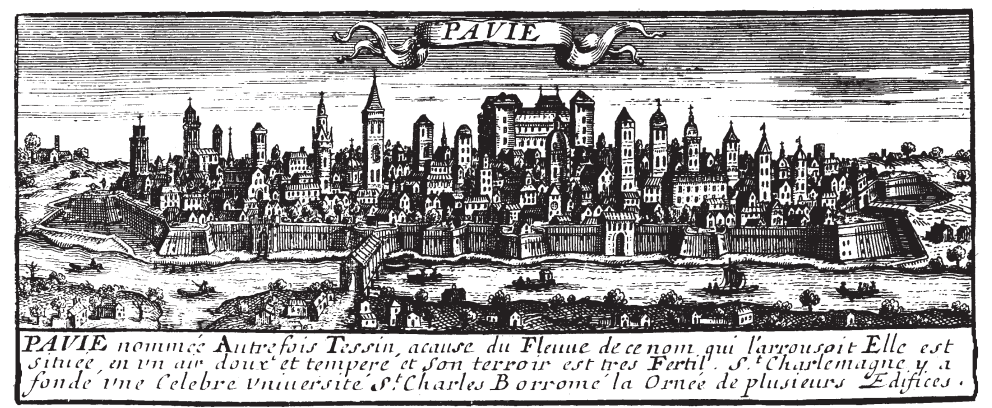

